\title{
Hepatitis, AIDS, and tuberculosis: hazards for anaesthetists
}

Arnold J. Berry MD

Over the past decade as the acquired immune deficiency syndrome (AIDS) epidemic has spread around the globe, the public and the medical community have become more aware of issues related to infection control. Health care workers (HCW) are increasingly cognizant of the risk of occupationally acquired diseases. Bloodborne pathogens such as human immunodeficiency virus (HIV), hepatitis B virus (HBV), hepatitis C virus (HCV), and respiratory transmitted pathogens such as tuberculosis, are now considered occupational hazards for HCW. Because of their contact with blood, body fluids, and respiratory secretions, anaesthetists are at high risk for infection transmitted by patients.

\section{Bloodborne pathogens}

In the community, bloodborne pathogens are transmitted between persons via several mechanisms including sexual contact, shared needles, or from mother to child. Other patients may acquire these infections through administration of contaminated blood or blood products. In contrast, occupational transmission to $\mathrm{HCW}$ involves percutaneous, mucous membrane, or non-intact skin exposure to infected patients' blood, blood-tinged fluids, blood products, tissues, or the following body fluids: semen, vaginal secretions, CSF, synovial fluid, pleural fluid, peritoneal fluid, pericardial fluid, or amniotic fluid. ' For anaesthetists, the majority of exposures are to blood or blood-tinged body fluids.

\section{Hepatitis B}

Before the development of the hepatitis B vaccine, occupational HBV infection was the greatest risk for health care workers. The US Centers for Disease Control and Prevention (CDC) estimates that occupational exposure to blood or body fluids results in 5,100 HBV seroconversions in $\mathrm{HCW}$ annually. ${ }^{2} \mathrm{~A}$ chronic $\mathrm{HBV}$ carrier state occurs after $10 \%$ of acute infections in adult patients. It is estimated that $21 \%$ of the chronic carriers or 170 HCW annually die of the long-term complications, cirrhosis or hepatocellular carcinoma. ${ }^{2}$ The risk of acquiring hepatitis B from a percutaneous exposure to blood from a patient carrying the HBV is up to $30 \%$.

Several serological surveys have demonstrated that anaesthetists are at high risk for acquiring HBV in the work- place. Multicentre prevalence studies of serological markers of HBV showed a range in US anaesthesia residents of $9-18 \%^{3}$ and in practicing anaesthetists and nurse anaesthetists of $19-49 \%{ }^{4,5}$ This is considerably greater than the 3-5\% found in the US general population as determined by screening healthy blood donors. Similar studies of anaesthesia personnel from other countries have found comparable values. ${ }^{5}$

The primary strategy for preventing $\mathrm{HBV}$ infection in $\mathrm{HCW}$ is the hepatitis $\mathrm{B}$ vaccine. ${ }^{6}$ The $\mathrm{CDC}$ has recommended that the vaccine be administered to all $\mathrm{HCW}$ that are exposed to blood and to medical, dental, and nursing students before their initial clinical rotations. Vaccines licensed for use in the US are derived from genetically engineered hepatitis $B$ surface antigen and carry no risk for transmission of HIV or other bloodborne pathogens. A three-dose regimen produces protective antibodies in about $95 \%$ of healthy adults. It is recommended that anaesthetists have their antibody status (anti-HBs) tested about six weeks after the final dose of vaccine to ensure effective immunity. Ongoing studies indicated that vaccine responders have protection against $\mathrm{HBV}$ infection for at least seven years and, at present, there is no recommendation for routine booster doses.

\section{Non-A, non-B hepatitis}

The majority of cases of parenterally transmitted non$\mathrm{A}$, non-B hepatitis are produced by $\mathrm{HCV}^{7,8}$ Based on this information, serological tests to detect antibody to HCV have been developed to assist in diagnosis. Unfortunately, not all patients with hepatitis $\mathrm{C}$ become antibody positive, and the test is not useful for identifying carrier status or chronic infection. Unlike antibody to HBV surface antigen, anti-HCV does not provide protective immunity. Approximately $50 \%$ of individuals infected with HCV develop chronic hepatitis.

The HCV is transmitted via mechanisms similar to other bloodborne infections. ${ }^{9}$ Although $90 \%$ of cases of post-transfusion hepatitis are non-A, non-B and presumably due to HCV, only about $6-10 \%$ of cases of acute

From the Department of Anesthesiology, Emory University School of Medicine, Atlanta, Georgia 30322. 
non-A, non-B hepatitis result from transfusion. Occupational transmission of HCV has been documented after percutaneous exposure to HCV-infected blood. The rate of transmission is $3 \%$ after a $\mathrm{HCV}$-contaminated needlestick injury. Healthcare workers account for $2-3 \%$ of all cases of non-A, non-B hepatitis.

At present there is no vaccine for immunization against $\mathrm{HCV}$. Immune gamma globulin prophylaxis is no longer recommended after a percutaneous exposure to $\mathrm{HCV}$.

\section{HIV}

The spectrum of HIV infection ranges from the asymptomatic, infected phase to AIDS with its associated opportunist infections and malignancies. It is estimated that worldwide, there are 14 million people infected with HIV with one-million in the US. Through June 1994, over 401,000 cases of AIDS in the US have been reported to the CDC. ${ }^{10}$

Several lines of evidence have been used to determine the occupational risk of HIV infection in HCW. A prospective study by the CDC has documented four seroconversions after 1,167 percutaneous exposures $(0.34 \%)$ to blood or body fluids from HIV-infected patients. "I This study reported no seroconversions after exposures to mucous membranes or intact skin. As of June 1994, there have been $42 \mathrm{HCW}$ documented by the CDC to have HIV infection after occupational exposure. ${ }^{10}$ The majority of these resulted from exposure to HIV-positive blood. Thirty-six were percutaneous exposures, four had mucocutaneous exposure, and one had both. These studies indicate that the greatest risk of occupational transmission HIV to $\mathrm{HCW}$ is through percutaneous exposures, with most of these being needlestick injuries. Analysis suggests that injuries with blood-filled, hollow-bore needies are associated with the greatest risk of transmission. This may be related to the larger innoculum from the greater volume of blood carried by this type of needle.

Because of procedures performed by anaesthetists, they are at risk for needlestick injuries, and many of the devices used are blood-filled, hollow-bore needles. ${ }^{12,13}$ The factors that determine the annual risk of acquiring HIV infection via a needlestick injury include: (1) the risk of seroconversion with each exposure to HIV-infected blood $(0.3 \%)$, (2) the prevalence of HIV-positive patients in the surgical population in the specific practice, and (3) the number of exposures per year. ${ }^{5,14}$ of these, the only variable that can be controlled is the number of exposures. Therefore, strategies to prevent occupational transmission of bloodborne infections have concentrated on eliminating exposures.

\section{Universal precautions}

The CDC has recommended the use of universal pre- cautions with all patients to reduce HCW exposure to bloodborne pathogens. ${ }^{1,15}$ Since all patients carrying bloodborne pathogens are not identified by the usual preoperative assessment, all patients should be treated with the same level of caution. Universal precautions include policies to prevent needlestick injuries and this includes prohibiting recapping of contaminated needles and recommendations for the use of puncture-resistant containers located in sites where needles are used. Barriers such as gloves, gowns, masks, and eye protection should be used when contact with blood or body fluids is anticipated. Health care workers with open skin lesions should avoid patient contact since the non-intact skin provides a portal of entry for bloodborne viruses. Since gloves may have unrecognized tears, hand washing is recommended after removal of gloves or as soon as possible after cutaneous exposure to infected material.

In 1992, the U.S. Occupational Safety and Health Administration (OSHA) implemented a federal standard to protect employees against occupational exposure to bloodborne pathogens. ${ }^{16}$ This regulation requires employers to institute practices to ensure safe conditions for their employees. The primary strategy for reducing exposures to blood and body fluid is engineering controls which either remove the hazard or isolate the individual from exposure. Examples of this include needleless or protected needle devices to replace the standard sharps. Work practice controls reduce the risk of exposure by altering the way in which a task is performed. For example, an instrument should be used to grasp a needle rather than removing the needle from a syringe by hand. Additional safety devices and new methods of performing tasks associated with exposures must be sought.

\section{Cost of universal processions}

When the OSHA Standard was implemented, it was estimated that the overall annual cost of compliance in the US would be $\$ 813$ million. ${ }^{16}$ For all hospitals, the cost would be $\$ 322$ million annually or $\$ 51,950$ per hospital. A 900-bed university hospital in the US reported that their annual expenditure for universal precaution was $\$ 350,900$ with the majority being spent for gloves and gowns. ${ }^{17}$

A 400-bed acute care teaching hospital in Canada has reported the incremental cost of universal precaution to be $\$ 315,000$ per year. ${ }^{18}$ If it is assumed that the risk of seroconversion after an HIV-contaminated needlestick injury is $1 \%$ (a high estimate) and the hospital population has a prevalence of HIV-infected of patients of $1 \%$, the cost of universal precautions would be about $\$ 8$ million per case of HIV seroconversion prevented. As new technology and engineering controls are considered for use to prevent bloodbome infections, cost-effectiveness analyses must be performed. ${ }^{19}$ 


\section{Tuberculosis}

Although the number of cases of tuberculosis in the US had been steadily declining, the trend was reversed in 1985 with the rate of new cases increasing by $18 \%{ }^{20}$ In 1992, over 2,600 new cases of tuberculosis were reported to the CDC. Data from 1993 indicated a slight decrease in the number of US cases, but further information is necessary to determine whether this represents a trend towards further decline.

The prevalence of cases of tuberculosis with drugresistant organisms has increased. In New York City, 33\% of new cases of tuberculosis were resistant to isoniazid. One cause of drug-resistant organisms has been incomplete antibiotic treatment in some patient populations.

Since tuberculosis is transmitted through the air via droplet nuclei, $\mathrm{HCW}$ are at risk for infection from patients with active pulmonary disease. ${ }^{21}$ Because of frequent contact with the airway, anaesthetists are at high risk when caring for patients with active tuberculosis. Effective infection control for tuberculosis involves early identification of infected patients and respiratory isolation of those suspected of having tuberculosis. Isolation must be continued during evaluation of the patient and until there has been documented efficacy of treatment by negative sputum cultures and smears as well as resolution of symptoms. Respiratory isolation consists of the use of negative pressure rooms, use of masks by patients when out of their isolation room, and personal particulate respirators for HCW in situations when the patient cannot wear a mask. Elective surgery should not be performed on patients with active disease. Hospitals should have regular skin-testing programmes for $\mathrm{HCW}$ at risk for tuberculosis.

\section{References}

1 Anonymous. Guidelines for prevention of transmission of human immunodeficiency virus and hepatitis B virus to health-care and public safety workers. MMWR Morb Mortal Wkly Rep 1989; 38(Suppl.6): 1-37.

2 Short LJ, Bell DM. Risk of occupational infection with blood-borne pathogens in operating and delivery room settings. Am J Infect Control 1993; 21: 343-50.

3 Berry AJ, Isaacson IJ, Kane MA, et al. A multicenter study of the epidemiology of hepatitis $B$ in anesthesia residents. Anesth Analg 1985; 64: 672-6.

4 Berry AJ, Isaacson IJ, Kane MA, et al. A multicenter study of the prevalence of hepatitis B viral serologic markers in anesthesia personnel. Anesth Analg 1984; 63: 738-42.

5 Berry AJ, Greene ES. The risk of needlestick injuries and needlestick-transmitted diseases in the practice of anesthesiology. Anesthesiology 1992; 77: 1007-21.

6 Anonymous. Protection against viral hepatitis. Recom- mendation of the Immunization Practices Advisory Committee (ACIP). MMWR Morb Mortal Wkly Rep 1990; 39(RR-2): 1-26.

7 Alter HJ, Purcell RH, Shih JW, et al. Detection of antibody to hepatitis $C$ virus in prospectively followed transfusion recipients with acute and chronic non-A, non-B hepatitis. N Engl J Med 1989; 321: 1494-500.

8 Alter MJ. Hepatitis C. A sleeping giant? Am J Med 1991; 91(Suppl 3B): 112S-5S.

9 Alter MJ, Hadler SC, Judson FN, et al. Risk factors for acute non-A, non-B hepatitis in the United States and association with hepatitis $C$ virus infection. JAMA 1990; 264: 2231-5.

10 Anonymous. HIV/AIDS Surveillance Report. MMWR Morb Mortal Wkly Rep 1994; 6: 1-23.

11 Marcus $R$, and the CDC Cooperative Needlestick Surveillance Group. Surveillance on health care workers exposed to blood from patients infected with the human immunodeficiency virus. N Engl J Med 1988; 319: 1118-23.

12 Kristensen MS, Sloth E, Jensen TK. Relationship between anesthetic procedure and contact of anesthesia personnel with patient body fluids. Anesthesiology 1990; 73: 619-24.

13 Berry AJ. The use of needles in the practice of anesthesiology and the effect of a needleless intravenous administration system. Anesth Analg 1993; 76: 1114-9.

14 Buergler JM, Kim R, Thisted RA, Cohn SJ, Lichtor JL, Roizen MF. Risk of human immunodeficiency virus in surgeons, anesthesiologists, and medical students. Anesth Analg 1992; 75: 118-24.

15 Anonymous. Update: Universal precautions for prevention of transmission of human immunodeficiency virus, hepatitis $B$ vinus, and other bloodborne pathogens in health-care settings. MMWR Morb Mortal Wkly Rep 1988; 37: 377-82.

16 Occupational Exposure to Bloodborne Pathogens; Department of Labor, Occupational Safety and Health Administration. Final Rule (29 CFR Part 1910.1030). Federal Register 1991; 56: 64005.

17 Doebbeling $B N$, Wenzel RP. The direct costs of universal precautions in a teaching hospital. JAMA 1990; 264: 2083-7.

18 Stock SR, Gafni A, Bloch RF. Universal precautions to prevent HIV transmission to health care workers: an economic analysis. Can Med Assoc J 1990; 142: 937-46.

19 Laufer FN, Chiarello LA. Application of cost-effectiveness methodology to the consideration of needlestick-prevention technology. Am J Infect Control 1994; 2: 75-82.

20 Anonymous. Tuberculosis morbidity - United States, 1992. MMWR Morb Mortal Wkly Rep 1993; 42: 696-7.

21 Dooley SW Jr, Castro KG, Hutton MD, Mullan RJ, Polder $J A$, Snider $D E J r$. Guidelines for preventing the transmission of tuberculosis in health-care settings, with special focus on HIV-related issues. MMWR Morb Mortal Wkly Rep 1990; 39(no. RR-17): 1-29. 


\section{L'hépatite, le SIDA et la tuberculose: des menaces pour l'anesthesiste}

Au cours de la dernière décennie, à mesure que le syndrome dimmuno-déficience acquise (SIDA) s'étendait mondialement, le public et la communauté médicale ont senti de besoin d'approfondir leurs connaissances sur les sujets relatifs au contrôle de l'infection. Les travailleurs de la santé comprennent de mieux en mieux le risque des maladies acquises au travail. Les pathogènes transportés par le sang comme le virus d'immuno-déficience humain (VIH), de l'hépatite B (VHB), le virus de l'hépatite $\mathrm{C}$ (VHC), et des pathogènes transmis par la respiration comme la tuberculose, sont maintenant reconnus comme des maladies professionnelles pour les travailleurs de la santé. A cause des contacts qu'ils ont avec le sang, les liquides organiques et les sécrétions respiratoires, les anesthésistes présentent un risque élevé pour les infections transmises par leurs patients.

\section{Les pathogènes transportés par le sang}

Dans le public en général, les pathogènes sanguins sont transmis entre les personnes par plusieurs mécanismes dont les contacts sexuels, le partage des aiguilles, et par la communication entre la mère à l'enfant. Les patients peuvent attraper ces infections par l'administration de sang ou de produits sanguins contaminés. Par contre, la transmission au travail dans le milieu médical implique un contact par la peau, les membranes muqueuses ou des contacts non cutanés avec le sang de patient infectés, avec des liquides teintés de sang, des produits sanguins, des tissus ou encore d'autres liquides organiques: le sperme, les sécrétions vaginales, le liquide céphalorachidien, le liquide synovial, le liquide d'épanchement pleural, le liquide péritonéal, péricardique ou amniotique. ${ }^{1}$ Pour les anesthésistes, la majorité des contacts se font par le sang ou des liquides teintés de sang.

\section{L'hépatite B}

Avant que se soit développé le vaccin contre l'hépatite $B$, le risque d'une infection professionnelle par le VHB était très élevé chez les travailleurs de la santé. Le centre américain pour le contrôle et la prévention de la maladie (Center for Disease Control: CDC) estime que, dans le milieu de travail, l'exposition au sang et aux liquides or- ganiques entraine annuellement 5100 séroconversions au VHB chez les travailleurs de la santé. ${ }^{2}$ Dix p. cent des adultes infectés de façon aiguë par le VHB deviennent porteurs chroniques. On estime que, par année, $21 \%$ des porteurs chroniques, ou 170 travailleurs de la santé, décéderont de complications à long terme comme la cirrhose ou le cancer hépatocellulaire. ${ }^{2}$ Le risque de contracter l'hépatite B lors d'un contact percutané avec le sang d'un malade porteur du VHB est de plus de $30 \%$.

Plusieurs enquêtes sérologiques ont révélé que les anesthésistes sont à haut risque d'acquérir le VHB dans leur milieu de travail. Aux Etats-Unis, des études multicentriques réalisées avec les marqueurs sérologiques du VHB ont montré que la prévalence chez les résidents en anesthésie se situe entre $9 \%$ et $18 \%{ }^{3}$ et chez les anesthésistes et les infirmières anesthésiste, entre $19 \%$ et $49 \% .^{4,5} \mathrm{Ceci}$ est évidemment beaucoup plus que les $3 \%$ et $5 \%$ rencontrés dans la population américaine totale comme le montre le dépistage chez des donneurs de sang en bonne santé. En anesthésie, des études identiques provenant d'autres pays et portant sur le personnel mis ont en évidence des valeurs comparables. ${ }^{5}$

Pour prévenir les infections à l'hépatite $\mathrm{B}$ chez les travailleurs de la santé, la stratégie fondamentale consiste à vacciner contre le VHB. ${ }^{6}$ Le CDC recommande que le vaccin soit administré à tous les travailleurs de la santé qui sont exposés au sang et à tous les étudiants en médecine, dentisterie, ou soins infirmiers avant leur premier stage clinique. Les vaccins reconnus pour usage au EtatsUnis sont dérivés de l'antigène de surface de l'hépatite B manipulé génétiquement et ne comportent pas de risque de transmission du VIH ou de tout autre pathogène véhiculé par le sang. Un ensemble de trois injections produit des anticorps protecteurs dans environ $95 \%$ des adultes en bonne santé. Il est recommandé aux anesthésistes de faire analyser leur concentration d'anticorps environ six semaines avant la dernière dose du vaccin pour s'assurer d'une immunité complète. Des études en cours indiquent que ceux qui répondent au vaccin ont une protection contre linfection au VHB pour au moins sept ans et jusqu'à maintenant, aucune recommandation favorisant les doses de rappel de routine n'a été avancée. 


\section{L'hépatite non-A, non-B}

La majorité des cas d'hépatite non-A, non-B transmise par voie parentérale sont causés par le VHC. ${ }^{7,8}$ A partir de cette donnée, les tests sérologiques pour détecter les anticorps au VHC ont été développés pour aider au diagnostic spécifique. Malheureusement, tous ceux qui possèdent des anticorps positifs pour l'hépatite $\mathrm{C}$ ne deviennent pas VHC-positifs et le test ne vaut pas pour lidentification des porteurs ou des infectés chroniques. Contrairement à l'anticorps ou à l'antigène de surface VHB, l'anti-VHC ne possède pas de propriété immunitaire. Environ $50 \%$ des individus infectés par le VHC développent une hépatite chronique.

Le VHC est transmis par les mécanismes semblables à ceux des autres infections véhiculées par le sang. ${ }^{9}$ Bien que $90 \%$ des cas d'hépatite post-transfusionnelle soient non-A, non-B et donc présumés dus au VHC, seulement 6 à $10 \%$ des cas d'hépatite aiguë non- $A$, non-B résultent de transfusions. La transmission au travail du VHC après une exposition percutanée à du sang infecté par le VHC a été documentée. Le taux de transmission est de $3 \%$ après une blessure par aiguille contaminée par le VHC. Les travailleurs de la santé représentent 2 à $3 \%$ de tous les cas d'hépatite non-A, non-B. Au moment présent, il n'existe pas de vaccin pour immuniser contre le VHC. Après une exposition percutanée au VHC, la prophylaxie à la gammaglobuline immune n'est plus recommandée.

\section{Le VIH}

La fourchette des infections au VIH s'étend de la phase asymptomatique de l'infection jusqu'au SIDA avec infections opportunistes et malignité. Sur notre planète, on croit que 14 millions de personnes sont infectées par le VIH; un demi-million le sont aux État-Unis. En juin 1994, plus de 401,000 cas de SIDA ont été rapportés au CDC. ${ }^{10}$

Plusieurs filières ont été utilisées pour déterminer le risque de transmission au travail de linfection au VIH chez les travailleurs de la santé. Une étude prospective menée par le $\mathrm{CDC}$ a documenté quatre séroconversions après 1167 cas de contacts percutanés $(0,34 \%)$ avec la sang ou les liquides organiques provenant de patients infectés par le VIH." Cette étude ne rapportait aucune séroconversion après exposition au HIV des membranes muqueuses et de la peau intactes. Jusqu'en juin 1994, le CDC a fait la preuve que 42 travailleurs de la santé ont acquis des infections au VIH après une exposition dans leur milieu de travail. ${ }^{10}$ La majorité de ceux-ci résultait d'une exposition à du sang VIH-positif. Trente-six des cas d'infection résultaient d'un contact percutané, quatre de contacts mucocutanées et un provenait des deux. Ces études montrent que pour les travailleurs de la santé, le plus grand risque de transmission du VIH dans leur milieu de travail, provient des expositions percutanées et dont la plupart sont des blessures par piqûres d'aiguilles. L'analyse suggère que les blessures provenant d'aiguilles creuses remplies de sang sont associées avec le plus grand risque de transmission. Ceci peut s'expliquer par l'abondance de linnoculum contenu dans le sang provenant de ces aiguilles.

A cause de leurs modes d'interventions, les anesthésistes sont plus à risque de blessures par piqûres d'aiguilles, et plusieurs des dispositifs quilils utilisent, dont les aiguilles creuses, sont remplis de sang. ${ }^{12,13}$ Les facteurs qui déterminent le risque annuel de contracter d'une infection au VIH par piqûre d'aiguille sont les suivants: premièrement, le risque de séroconversion à chaque exposition au sang infecté par le VIH $(0,3 \%)$; deuxièmement, la prévalence de patients VIH-positifs dans la population où on exerce; et troisièmement, le nombre d'expositions par année. 5,14 La seule variable contrôlable parmi ces facteurs est le nombre d'expositions. De là, les stratégies de prévention au travail de la transmission d'infections véhiculées par le sang se sont concentrées sur l'élimination des contacts.

\section{Les précautions universelles}

Le CDC recommande l'usage de précautions intégrales en présence de tous les patients dans le but de diminuer l'exposition des travailleurs de la santé aux pathogènes transportés par le sang. ${ }^{1,15}$ Comme, en général, les patients porteurs de pathogènes transmis par le sang ne sont pas décelables par les évaluations préopératoires usuelles, tous doivent être abordés avec le même degré de prudence. Les précautions intégrales devraient inclure des politiques de prévention des blessures par pigûre d'ajguille et ceci inclut le recapuchonnage des aiguilles souillées et des recommandations pour lutilisation de réceptables résistants aux ponctions, situés là où les aiguilles sont utilisées. Des écrans tels que gants, blouses, masques, lunettes devraient être utilisés quand on anticipe un contact avec le sang ou les liquides corporels. Les travailleurs de la santé qui souffrent de lésions cutanées ouvertes devraient éviter tout contact avec les patients parce qu'une lésion de la peau fournit une porte d'entrée pour les virus transportés par le sang. Comme les gants peuvent porter des déchirures occultes, le lavage des mains est recommandé lorsque les gants sont retirés ou aussitôt que possible après une exposition cutanée à du matériel infecté.

En 1992, l'OSHA (Occupational Safety and Health Administration) a implémenté un standard fédéral pour protéger les employés contre les expositions au travail à des pathogènes transportés par le sang. ${ }^{16} \mathrm{Ce}$ règlement requiert que les employeurs instituent des méthodes dans le but assurer les meilleures conditions de sécurité pour 
leurs employés. La principale stratégie recommandée pour diminuer l'exposition au sang et aux liquides corporels consiste à développer des contrôles qui suppriment le risque ou protègent l'individu exposé. Par exemple, on peut utiliser des dispositifs qui fonctionnent sans aiguille ou des aiguilles mousses pour remplacer les aiguilles aiguisées habituelles. Le contrôle des habitudes de travail diminue le risque d'exposition si on modifie la façon d'accomplir une tâche: par exemple, utiliser une pince pour saisir l'aiguille au lieu d'enlever l'aiguille avec ses doigts. D'autres dispositifs de sécurité, et de nouvelles méthodes pour réaliser une tâche associée à une contact possible avec le pathogène doivent être trouvées.

\section{Coût des procédés de protection universelle}

Quand le standard de OSHA a été mis en vigueur aux Etats-Unis, on a estimé que le coût annuel total de son implémentation serait de $\$ 813$ million. ${ }^{16}$ Pour tous les hôpitaux, le coût devait être annuellement de $\$ 322$ millions ou encore de $\$ 51950$ par hôpital. Un hôpital universitaire de 900 lits a rapporté que ses dépenses totales encourues pour les précautions intégrales étaient de $\$ 350900$ dont la majorité avait servi pour l'achat de champs et de blouses.

$\mathrm{Au}$ Canada, un hôpital d'enseignement consacré aux soins aigus a rapporté que le coût de limplémentation par pallier des précautions universelles était de $\$ 315000$ par année. ${ }^{18} \mathrm{Si}$ on assume que le risque de séroconversion après une blessure par piqûre d'aiguille contaminée par le VIH est de $1 \%$ (l'estimé le plus élevé) et que la population hospitalière a une prévalence de patients infectés par VIH de $1 \%$, le coût des précautions universelles serait de l'ordre de $\$ 8$ millions par cas de prévention de séroconversion du VIH. A mesure qu'on envisage de nouveaux contrôles technologiques pour la prévention des infections transmises par le sang, les analyses du coût par rapport à l'efficacité doivent être réalisées. ${ }^{19}$

\section{La tuberculose}

Bien que le nombre de cas de tuberculose aux EUA ait décliné constamment, la tendance a été renversée en 1985 avec un taux d'augmentation de $18 \% .{ }^{20}$ En 1992, plus de 26000 nouveaux cas de tuberculose ont été rapportés au CDC. Les données de 1993 révèlent une légère diminution du nombre de cas aux EUA, mais nous avons besoin d'accumuler plus de données pour déterminer si cette diminution représente une nouvelle tendance.

La prévalence des cas de tuberculose résistants aux médicaments a augmenté. Dans la ville de New York, $33 \%$ des nouveaux. cas de tuberculose sont résistants à l'isoniazide. Une des cause de la résistance des organismes aux médicaments serait l'insuffisance du traitement aux antibiotiques pour une partie de la population.
Comme la tuberculose est transmise dans l'air par des gouttelettes, les travailleurs de la santé risquent d'être infectés par des patients atteints dune maladie pulmonaire active. ${ }^{21}$ A cause de leur contact fréquent avec les voies respiratoires, les anesthésistes assument un risque lorqu ils s'occupent de tuberculeux actifs. Le contrôle efficace de l'infection nécessite une identification précoce des patients infectés et l'isolation respiratoire de ceux qu'on suspecte de tuberculose. Lisolation doit être maintenue pendant l'évaluation et jusqu'à ce qu'on ait prouvé l'efficacité du traitement par des cultures et des frottis d'expectorations négatifs, aussi bien que par la résorption des symptômes. L'isolement du système respiratoire se fait par lutilisation de chambres à pression négative, du port de masque par le patient quand ils circulent en-dehors de leur chambre d'isolement, et un masque spécial pour les travailleurs de la santé dans les situations oû le patient n'en porte pas. Une chirurgie non urgente ne devrait pas être réalisée chez les porteurs de tuberculose active. Les hôpitaux devraient favoriser la réalisation d'épreuves cutanées régulières pour les travailleurs de la santé à risque de tuberculose.

\section{Références \\ (Voir page R16)}

